\title{
Adults with Neurodevelopmental Disorders Continue to Receive Lower Quality Health Services: A Call for "Quality Improvement" Programs
}

\author{
Luz Fernandez, $M D^{1}$, Jon Becker, $M D^{2}$ and Philip May, $M D^{3 *}$
}

${ }^{1}$ Director, Family Medicine Residency Training Program, Associate Professor of Family Medicine, University of Louisville School of Medicine, USA

${ }^{2}$ Chief, Department of Family Medicine \& Geriatrics, Professor of Family Medicine, University of Louisville School of Medicine, USA

${ }^{3}$ Director of Quality Improvement Program (QIP) Development, International Foundation for Chronic Disabilities (IFCD), Inc., USA

*Corresponding author: Philip May, Director of Quality Improvement Program (QIP) Development, International Foundation for Chronic Disabilities, Inc.; Clinical Associate Professor of Medicine (Gratis), University of Louisville School of Medicine, PO Box 16057, Louisville, KY 40256, USA

\begin{abstract}
Neurodevelopmental Disorders (also known as Intellectual and Developmental Disabilities-ID/DD) are associated with multiple-complex-health-conditions that, in contrast to children, are often not evaluated and/or managed properly as adults. The specialty of Developmental and Behavioral Pediatrics exists for children with ID/DD, but there is no equivalent specialty for the adults. Since Family Medicine has taken the lead in providing primary care for adults with ID/DD, we propose that Family Medicine follow the example of Pediatrics by organizing multisite University-based Quality Improvement (QI) programs in Family Medicine Departments (with Residents, Students, and Fellows). To begin the process and create focus, the specific problem of obesity (and its complications) could be addressed in the adults utilizing Quality Improvement methods designed to promote teaching, research, and improved health outcomes. Participating University sites could then organize to create a new academic professional organization focused on all health-issues of adults with ID/DD, as occurred in Pediatrics for children.
\end{abstract}

\section{Introduction}

"Neurodevelopmental disorders" (NDD), also known as Intellectual and Developmental Disabilities (ID/DD), are genetic or acquired chronic health conditions that interfere with various functions of the brain (Figure 1). These conditions persist into adulthood and are responsible for 4 categories of "disabilities"; namely, mild, moderate, or severe 1) Cognitive dysfunction, 2) Motor dysfunction, 3) Seizures, and/or 4) Maladaptive behavior (Figure 2). These four categories of health conditions, or "complications", can occur singly or in various combinations and may also lead to numerous secondary health "consequences" (e.g., obesity, osteoporosis, drug toxicity, etc.). In addition, there may also be complications specific for a syndrome, unrelated to brain dysfunction $[1,2]$.

At least since the 1800's [3], physicians have recognized the complexity of health conditions experienced by adults with NDD. In the United States, an Accreditation Council for Graduate Medical Education (ACGME) approved medical specialty (Developmental and Behavioral Pediatrics) which addresses these health conditions in children has existed in the United States since 1999 [4]. Unfortunately, there are no analogous programs designed to train specialist physicians in the research, teaching, and service of health-conditions of adults with neurodevelopmental disorders [59]. Thus, in the United States, when children with

Citation: Fernandez L, Becker J, May P (2021) Adults with Neurodevelopmental Disorders Continue to Receive Lower Quality Health Services: A Call for “Quality Improvement" Programs. Int J Brain Disord Treat 6:039. doi.org/10.23937/2469-5866/1410039

Accepted: November 13, 2021: Published: November 15, 2021

Copyright: (C) 2021 Fernandez L, et al. This is an open-access article distributed under the terms of the Creative Commons Attribution License, which permits unrestricted use, distribution, and reproduction in any medium, provided the original author and source are credited. 


\section{A Brief Classification of "Neuro-developmental disorders"}

1. "Genetic" Disorders (abnormal gene/genes):

A. Metabolic: Missing enzyme leads to build-up of toxic metabolite (secondary brain damage) and/or deficiency of essential end product needed for neuronal function (primary brain damage).

"Primary" metabolic causes:

I. Lipids: Sulfatide-lipidosis (Metachromatic Leukodystrophy)

2. Mucopolysaccharides: (Sanfilippo Syndrome)

"Secondary" metabolic causes

I. Amino Acids: Phenylalanine accumulation (Phenylketonuria), Homocysteine accumulation (Homocystinuria)

2. Purines/pyrimidines: Uric acid accumulation (Lesch-Nyhan Syndrome)

B. Structural Malformations: Cellular derangement or malfunction of brain tissue, usually with structural abnormalities of the brain and often other body parts. No known enzyme deficiency or chromosomal abnormality.

1. Neurodysplasia:

a. Tuberous Sclerosis; b. Neurofibromatosis

2. Neuro-dysgenesis: neurulation, prosencephalic segmentation, neuronal proliferation, neuronal migration, organization, myelination:

a. Lissencephaly (some types); b. Schizencephaly (some types) c. Septo-Optic Dysplasia (some types)

3. Unknown:

a. Sturge Weber Syndrome; b. Rett Syndrome-MEC-P2 mutation

C. Chromosomal Abnormalities:

1. Triplet Repeat Disorders

a. Fragile X Syndrome (C-X); b. Myotonic Dystrophy (C-19)

2. Aneuploidies:

a. Down syndrome; b. Trisomy 8 Mosaic Syndrome

c. Trisomy 13; d. Trisomy 18

3. Microdeletions:

a. William's Syndrome (C-7)

b. Prader-Willi Syndrome (paternal C-15)

c. Angelman's Syndrome (maternal C-15)

d. Smith-Magenis Syndrome (C-17)

e. Rubenstein-Taybi Syndrome (C-16)

f. Velo-Cardio-Facial Syndrome (C-22)

4. Rearrangements:

a. Ring 22 syndrome

2. "Acquired" Disorders:

A. Prenatal Disorder

I. Infections (TORCH, Rubella)

2. Toxins (alcohol, maternal PKC)

3. Traumatic (ischemia-hypoxia)

4. Structural Malformations

a. Arnold-Chiari Malformation; b. Dandy-Walker Cyst

c. Agenesis of Corpus Callosum; d. Septo-Optic Dysplasia (some types)

e. Lissencephaly (some types)

B. Perinatal Disorder

I. Traumatic(hypoxia-ischemia); 2. Toxins (Kernicterus)

3. Prematurity/Low Birth Weight

C. Post-Natal Disorder

1. Infection (measles); 2. Traumatic; 3. Metabolic (hypothyroid)

4. Neoplasm; 5. Toxins (lead)

Figure 1: A brief classification of neuro-developmental disorders.

neurodevelopmental disorders are transitioned into adult services, there are no ACGME approved "Board Certified" specialists in adult Neurodevelopmental Medicine to continue the same high-quality health
Cause, Complications, \& Consequences of Childhood Onset Brain Dysfunction

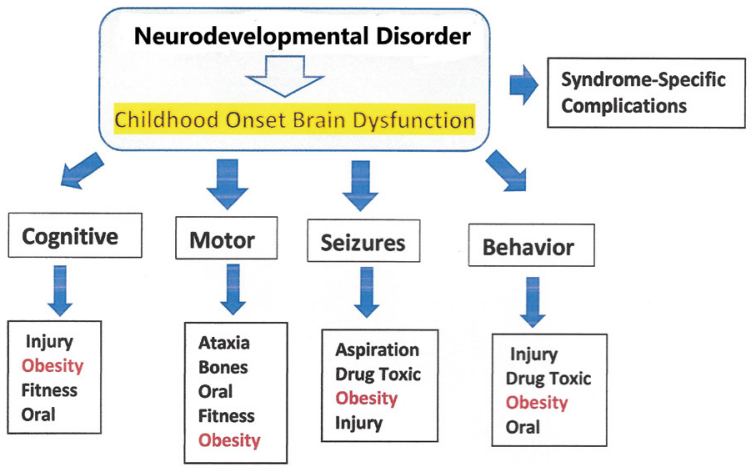

Figure 2: Cause, complications and consequences of childhood onset brain dysfunction.

services they received as children. A similar situation appears to exist internationally.

In the universities, there has been little interest in development of 1) University-based medical education [5] 2) "Quality Improvement" Programs [10] and/ or 3) Clinical research [11] designed to improve delivery of high-quality evidence-based healthcare for adults with NDD. Therefore, as of 2002, even though remarkable improvements had been achieved for social, non-medical community services (e.g., residential, employment, educational, recreational) modern generations of physicians who provide primary healthcare to adults (Family Physicians and Internists), remain untrained regarding comprehensive evaluation of health-conditions that often present in adults with neurodevelopmental disorders (e.g., obesity, osteoporosis, lack of genetic vs. acquired etiologic diagnosis, etc.) [12].

As former US Surgeon General David Satcher MD succinctly stated in the introduction to the historic Surgeon General's Report of his Conference in 2001, "as services for people with mental retardation evolved, our attention to their health lessened". Then US Secretary of Health and Human Services, Tommy G. Thompson, concluded in the same report, "too few providers receive adequate training in treating persons with mental retardation. Even providers with appropriate training find our current service system offers few incentives to ensure appropriate health care for children and adults with special needs. American health research, the finest in the world, has too often bypassed health and health services research questions of prime importance to persons with mental retardation" [11].

\section{Service for Children, a Model for the Adults}

While major healthcare-service deficiencies still exist for adults with neurodevelopmental disorders, healthcare-services for children with neurodevelopmental disorders, have significantly improved and how these improvements were achieved could serve as a model for improvement of healthcare 
quality for adults. In the United States, health issues of children with neurodevelopmental disorders greatly benefited from the work of the Society for Developmental and Behavioral Pediatrics (SDBP) (SDBP Website) [4]. Formation of the SDBP initially began in 1976 by eleven University-based Directors of Training Grants provided by the W.T. Grant Foundation of New York City. The purpose of those training grants was to train Pediatric Residents in how to better care for children with ID/DD. After meeting yearly for 5 years, from 1977-1982 (required as a condition of funding), these eleven Medical School-based training Directors (all University Professors of Pediatrics) and colleagues formally created the SDBP for the purposes of continuing to exchange ideas and to encourage research in the field by presenting research papers at its annual meeting, establishing additional training programs, and creation of a Society journal. In addition, because of the advocacy of the Society (SDBP website), since 1986 the U.S. Maternal and Child Health Bureau of HRSA has provided funding for 3-year Universitybased research/academic Fellowship Programs in the field of Developmental and Behavioral Pediatrics. In the United States, several hundred ACGME certified Board-Certified pediatric specialists in Developmental \& Behavioral Pediatrics now exist for children (SDBP website) [4].

Recognizing the deficiency of quality primary health services for adults with ID/DD, the discipline of Family Medicine has been comprehensively addressing this problem since 2011 [12]. Family Medicine has been providing culturally competent compassionate primary care, but due to lack of quality-improvement programs and clinical research, major deficiencies in the practice of evidence-based-medicine for adults with Intellectual Disability still exist. In addition, ACGME-certified Fellowships have not been established in Family Medicine for adults with ID/DD as they have been for children

\section{Adult "Quality Improvement" (QI) Programs: One Way to Begin}

To begin to eliminate these health-disparities, we propose that the specialty of Family Medicine follow the lead of Pediatrics. This could begin by government and/or private foundation funding of "Quality Improvement" [13,14] grants awarded to University Departments of Family Medicine, with the stipulation that Directors of these "Quality Improvement" grants meet regularly to share outcomes and to also establish training of medical students and residents and conduct "Quality Improvement" Programs which address health conditions of adults with neurodevelopmental disorders [12]. "Quality Improvement" programs are often implemented for general medical practices [14], but it is uncommon for the QI approach to be applied to the adult population of individuals with Neurodevelopmental Disorders [10]. Conditions such as obesity and osteoporosis are examples of health conditions that frequently occur in adults with neurodevelopmental disorders that could easily be addressed using QI methodology. Obesity is an especially important problem because of the associated higher risk of morbidity and mortality from cardiovascular, metabolic disorders, cancer, and many other problems [15]. In addition, there now appears to be an increased risk of poor outcome from COVID-19 infection associated with obesity [16], which may be worse in adults with ID/DD [17]. Developmental and Behavioral Specialist Pediatricians also have difficulty treating obesity in children [18]. If Obesity QI Programs were designed to include multiple University-based sites in a project which involves Medical Students, Residents, Fellows, and Faculty, valuable clinical information needed to improve health of obese adult participants with neurodevelopmental disorders could more readably be obtained. Furthermore, valuable information obtained from such QI Projects would lead to formal research projects designed to build a muchneeded evidence-base to promote optimal medical practice for adults with neurodevelopmental disorders.

\section{References}

1. May P (2006) The five essential concepts of developmental medicine: A new medical paradigm for persons with developmental disabilities. The Exceptional Parent 6: 6266 .

2. Rader R (2007) The emergence of the American academy of developmental medicine and dentistry: Educating clinicians about the challenges and rewards of treating patients with special health care needs. Pediatr Dent 29: 134-137.

3. Scheerenberger R (1983) A history of mental retardation. Paul H. Brookes Publishing Co.

4. Society for Developmental and Behavioral Pediatrics.

5. Keller S, Turek G, Asato M, Sanders JS, Margolis B, et al. (2020) Complexities of caring and transitioning care for patients with intellectual or developmental disability. Neurology Reviews: 124-131.

6. Kornblau BL (2014) The case for designating people with intellectual and developmental disabilities as a medically underserved population. ASAN Policy Brief.

7. Ankam NS, Bosques G, Sauter C, Steins S, Therattil M, et al. (2019) Competency-based curriculum development to meet the needs of people with disabilities: A call to action. Acad Med 94: 781-788.

8. Mysliwiec A, Saulicz E, Kuszewski M, Wolny TA, Knapik A, et al. (2015) Self-evaluation of the preparation of physicians and physiotherapists to provide medical services to people with intellectual disability. Journal of Intellectual \& Developmental Disability 40: 104-110.

9. Fenton SJ, Hood H, Holder M, May PB, Mouradian WE (2003) The American academy of developmental medicine and dentistry: Eliminating health disparities for individuals with mental retardation and other developmental disabilities. J Dent Educ 67: 1337-1344.

10. Edwards J, Mold F, Knivett D, Boulter P, Firn M, et al. (2018) Quality improvement of physical health monitoring 
for people with intellectual disabilities: An integrative review. J Intellect Disabil Res 62: 199-216.

11. US Surgeon General Report (2002) Closing the gap: A national blueprint to improve the health of persons with mental retardation: Report of the surgeon general's conference on health disparities and mental retardation.

12. Sullivan W, Diepstra H, Heng J, Ally S, Bradley E, et al. (2018) Primary care of adults with intellectual and developmental disabilities: 2018 canadian consensus guidelines. Can Fam Physician 64: 254-279.

13. US Department of Health \& Human Services-Health Resources and Services Administration (2011) Developing and implementing a QI plan.

14. Marjoua Y, Bozic KJ (2012) Brief history of quality movement in us healthcare. Curr Rev Musculoskelet Med 5: 265-273.
15. Segula D (2014) Complications of obesity in adults: A short review of the literature. Malawi Med J 26: 20-24.

16. Abbas AM, Fahey SK, Fawzy AT, Salem AS, Shawky MS (2020) The mutual effects of covid-19 and obesity. Obesity Medicine 19: 100250.

17. Gleason J, Ross W, Fossi A, Blonsky H, Tobias J, et al. (2021) The devastating impact of covid-19 on individuals with intellectual disabilities in the united states. NEJM catalyst.

18. Roizen NJ, Ruch-Ross HS, Bauer NS, Nielsen BA, DeBattista A, et al. (2021) Developmental-behavioral pediatrics 13 years after the first board certification: Evolving subspecialty. J Dev Behav Pediatr 42: 83-90. 